Article available at nttp://www.parasite-journal.org or nttp://dx.dol.org/10.1051/parasite/20020913

\title{
CONTRIBUTION À l'ÉtUde de LA PESTE AU VIETNAM : HISTORIQUe et INVENTAIRE DES PUCES SIGNALÉES (INSECTA - SIPHONAPTERA) EN ZONES ANTHROPISÉES
}

\author{
BEAUCOURNU J.-C.*, SOUNTSOVA N.I.**, LY T.V.H.*** \& SOUNTSOV V.V.**
}

Summary : CONTRIBUTION TO THE STUdy ON Plague FROM VIETNAM HISTORICAL VIEWS AND LIST OF THE COLLECTED FLEAS (INSECTA SIPHONAPTERA) IN ANTHROPIC ZONES

Ten flea species are reported in anthropic zones of Vietnam. Xenopsylla vexabilis is also here included because of it has been involved in others plague's countries. Lentistivalius klossi is the only selvatic flea known as parasite of synanthropic rats. L. klossi bispiniformis (Li and Wang, 1958), first describe from Chinese specimens, is here synonymized (syn. nov.) with the nominal subspecies.

KEY WORDS : Vietnam, plague, synanthropic fleas.

\section{Résumé :}

Dix espèces de puces sont connues au Vietnam en zones anthropisées. Xenopsylla vexabilis est incluse dans cette liste en raison de son rôle dans d'autres foyers pesteux. La seule puce selvatique connue comme pouvant parasiter des rats synanthropes est Lentistivalius klossi, dont nous mettons en synonymie la sousespèce bispiniformis (Li et Wang, 1958) (syn. nov.) décrite de Chine.

MOTS CLÉS : Vietnam, peste, puces synanthropes.

\section{INTRODUCTION}

I a peste, classée maladie réémergente par l'OMS depuis 1994, est apparue pour la première fois dans le Sud-Vietnam en 1898, quatre ans donc après le début de la $3^{\text {ème }}$ pandémie. On ne dispose pas à cette date de données sur le Nord du pays (Tonkin) mais il est vraisemblable que la proximité du Yunnan, foyer donné comme invétéré, avait amené cette région à être déjà atteinte.

La maladie va toucher d'abord la Cochinchine, par voie maritime, puis très vite exploser en Annam avec, pour 1908 , un total de 2435 cas pour l'ensemble de l'actuel Vietnam (Tonkin inclus par conséquent), culminant en 1910 à 2528 (Gaide et Bodet, 1930). Ce sera l'acmé officiel de la peste dans ce pays. Depuis, en dehors d'une courte disparition de cette maladie à la fin des années 30 , donc avant que les "évènements" de la $2^{\text {ème guerre }}$ mondiale n'empêchent ou ne faussent toute recherche, la peste se maintient, sur un mode endémique, faisant de ce pays l'un des foyers actifs de la région orientale (Vélimirovic, 1972; relevés OMS en particulier n 71, 1996

\footnotetext{
* Laboratoire de Parasitologie et Zoologie appliquée, Faculté de Médecine, Avenue du Professeur Léon Bernard, 35043 Rennes Cedex, France.

** Severtzov Institute of Ecology and Evolution RAS, Leninsky prosp. 33, 117071 Moscou, Russie.

${ }^{* * *}$ Institute of Hygiene and Epidemiology, Tay Nguyen, Buonmathuot, Vietnam.

Correspondance: J.C. Beaucournu.
}

et $n^{\circ}$ 42, 2000). De 1985 à 1999 (OMS, op. cit.), 3623 cas ont été signalés avec des incidences très variables d'une année à l'autre, ce qui est d'ailleurs classique. En moyenne, un peu plus de 240 cas annuels sont déclarés. En dehors de quelques foyers afrotropicaux, de celui de l'Iran et de ceux de l'ex-URSS pour la région paléarctique, peu ont fait l'objet de recherches entomologiques réelles dans l'Ancien Monde. La faune des puces du Vietnam était particulièrement mal connue jusqu'à ces dernières années et il est certain que beaucoup reste à faire.

\section{RAPPEL HISTORIQUE}

L e début de la présence trançaise dans ce qui fut alors appelé l'Indochine, coïncida pratiquement "Bacillus pestis" par Yersin en 1894 et de celle de son vecteur "la puce" par Simond en 1898. Cette dernière année fut également celle de l'apparition du premier foyer "officiel" de peste en Cochinchine, nous l'avons dit. Yersin (1899) estime, à propos du foyer de Nhatrang en Annam $^{1}$, que ce fut sans doute la première percée de cette maladie vers le sud de la Péninsule puisqu'elle n'y avait aucun nom vernaculaire, argument qui nous parait très valable.

\footnotetext{
${ }^{1}$ Étant donnée la date de cette publication, Hénaff, cité par Fréville (1932), se tromperait donc en affirmant que les premiers cas sont de 1906
} 
On sait que ce fut l'expérimentation de Simond à Karachi en Inde qui déclencha une remarquable émulation dans l'étude et, a fortiori, l'identification des Siphonaptères. Les "Reports on Plague investigation in India" ne retenaient en 1907 que six espèces pour le foyer indien et ceci en dépit des remarques de Rothschild et de Jordan, les meilleurs spécialistes de l'époque. Ces auteurs démontrent que Xenopsylla cheopis (Rothschild, 1903), la "puce pâle" comme la désignait l'Advisoree Committee (1907) n'était pas "le" vecteur mais un complexe : à lui seul le genre Xenopsylla regroupe actuellement près de 80 espèces. Cet élargissement de nos connaissances concerna, plus ou moins vite, plus ou moins facilement, l'ensemble des Siphonaptères du globe, qui, d'environ 95 taxa valides en 1900 (Beaucournu et Launay, 1990), passèrent à 2523 en 1997 (Lewis, 1998), chiffre évidemment provisoire.

L'endémie pesteuse dans la sous-région indochinoise est sous l'influence des foyers "birmans" et du Yunnan. Si l'Indochine française était plus une région administrative que géographique (Gaide et Bodet, 1930), elle renfermait cependant les éléments essentiels de cette entité biogéographique qu'est cette sous-région (Traub, 1972c), à savoir les pays autonomes que sont actuellement le Cambodge, le Laos et le Vietnam. Rappelons toutefois que la Thaillande, plaque tournante de cette zone sur le plan biogéographique (Lekagul, 1988), le Myanmar (ex Birmanie) et l'extrême sud de la Chine la complètent.

Lorsque l'on aborde une étude bibliographique des problèmes pesteux, et plus précisément vectoriels, au Vietnam, on est amené à se référer à des travaux ${ }^{2}$ s'étalant de 1917 avec Cadet, à l'époque actuelle. Particulièrement jusqu'aux années 50, les dénominations géographiques de Tonkin, d'Annam et de Cochinchine sont régulièrement utilisées et peuvent égarer : schématiquement, le Tonkin correspond à la majeure partie de l'actuel Nord-Vietnam; l'Annam à toute la zone côtière du sud de l'estuaire du Fleuve Rouge au sud du Plateau de Lang Bian; la Cochinchine s'étend du Nord de Saïgon (Hochiminh-Ville) à la pointe de Camau, extrêmité de la péninsule indochinoise.

Les foyers pesteux qui motivèrent la majorité des enquêtes médicales, comme les rares prospections à visées épidémiologiques (cherchant donc à cerner les hôtes-réservoirs et les puces vectrices), se rapportent essentiellement à la zone côtière et au sud du pays. $\mathrm{Au}$ demeurant, peu d'auteurs étudient des espèces selvatiques (hôtes ou vecteurs). Les puces citées sont toujours $X$. cheopis et, éventuellement, les taxa ou genres synanthropes, Pulex et Ctenocephalides. Rosti-

\footnotetext{
${ }^{2}$ Sans omettre celui de Yersin, cité plus haut, qui vue la date où cet article fut écrit, ne peut que citer le mot "puce" et donner son adhésion aux travaux de Simond.
}

gayev et Grokovskaya (1966) sont les premiers à identifier, dans l'optique de la Peste, quelques autres espèces, la plupart d'ailleurs déjà citées et décrites par Jordan ou Jordan et Rothschild ( $c f$. infra). Cavanaugh et al. (1969) écrivent : "it has not, at this date, been possible to examine many fleas from wild rodents. Those fleas which have been examined have been $X$. cheopis". Dans ces mêmes temps cependant, Traub et son équipe, travaillant dans le sud-est asiatique sur l'épidémiologie du typhus murin et de la peste, récoltaient un matériel considérable; de nombreux taxa nouveaux sont évoqués dans la Monographie de Traub (1972a, b, c) mais la plupart, dont ceux pouvant concerner le Vietnam, ne furent pas décrits ${ }^{3}$.

Par conséquent, en dehors de déjà anciennes descriptions d'espèces alors nouvelles, descriptions à visée purement entomologique concernant cette région (Jordan 1931, 1939; Jordan et Rothschild, 1922), c'est avec les enquêtes de Suntsov et al. (1992a, b, 1995, 1997, 1999) et les articles de Beaucournu et Sountsov (1997, 1998a, b, 1999a, b) ${ }^{4}$ et Beaucournu et al. (2000) étudiant le matériel récolté par cette équipe, que la faune des siphonaptères du Vietnam a pu commencer à être évaluée.

L'essentiel des données taxonomiques à notre disposition, principalement sur les espèces de puces selvatiques, ayant déjà été publié (cf. supra : 19 sur 38 étaient nouvelles pour la Science ou pour le Vietnam), c'est uniquement des Siphonaptères des villes et zones anthropisées que nous parlerons ici.

\section{ÉTUDE FAUNISTIQUE}

\section{Famille Pulicidae}

\section{Pulicinae}

\section{1 - Pulex irritans Linné, 1758}

Dès 1917, Cadet signale cette puce, appelée à tort "Puce de l'Homme", du Sud-Annam, en région côtière : environs de Phan Rang essentiellement; cet auteur la considère comme peu fréquente : "on en voit parfois au cours des séances de vaccination où on est littéralement submergé par un peuple en guenilles".

Hérivaux et Toumanoff (1948b) la notent pour SaïgonCholon, dans des poussières récoltées dans des paillottes et plus rarement des maisons en dur : 12 exemplaires sur 6830 puces récoltées au cours de 17 mois consécutifs (1943-1944); aucune n'est trouvée sur les rongeurs et la musaraigne Suncus murinus Linné, 1766, domiciliaires.

\footnotetext{
${ }^{3}$ R. Traub est décédé le 21 décembre 1996.

${ }^{4}$ Suntsov et Sountsov sont deux orthographes différentes concernant, bien sûr, le même chercheur; Sountsov est celle adoptée, par cet auteur russe, pour ses articles en français.
} 
Hopkins et Rothschild (1953) dans leur catalogue ne donne qu'une station dans le Sud-Annam, sur Homo. Rostigayev et Grokovskaya (1966), au Nord Vietnam la citent sur rongeurs (hôtes aberrants) : Mus musculus L., 1758 et Rattus flavipectus ( $=$ R. tanezum $i$ Temminck, $1844)^{5}$.

Enfin, Suntsov et al. (1992a) notent sa présence sur le plateau de Tay Nguyen, province de Dak Lak. Apparemment, elle existe dans tout le pays.

Il est vraisemblable que la prévalence de cette puce demeurera inconnue tant que son hôte selvatique, s'il existe dans cette sous-région biogéographique restera à trouver (son existence est très improbable en zone afrotropicale : Beaucournu et al., 1993). L'expérience de l'un de nous (J.C. B.) tant en Europe de l'Ouest, qu'en Afrique ou en Asie, montre, de plus, que l'étude des ectoparasites humains pose de nombreux problèmes éthiques, souvent insurmontables dans la pratique courante.

Son occurrence sur le chien "domestique" est normale, $P$. irritans est primitivement, nous le rappelons, une puce de canidés. Beaucournu et al. (2001) ne la rencontrent pas cependant dans le Nord-Laos ni sur l'homme ni sur le chien.

Matériel examiné6 $: 78$, provenant de chiens et du sol des cases.

Archaeopsyllinae

2 - Ctenocephalides canis (Curtis, 1826)

Cette espèce fut à tort ( $c f$. Beaucournu et Ménier, 1998) signalée à de nombreuses reprises du sud-est asiatique : il s'agissait en fait de C. orientis (Jordan, 1925). Cadet (1917) fait, par exemple, état de sa présence au Sud-Annam : la confusion était à cette époque inévitable. Hérivaux et Toumanoff (1948a, b), quant à eux, ne séparent pas, dans leurs tableaux, $C$. felis de C. canis bien qu'ils fassent référence à ces deux espèces. Il ne fait pour nous aucun doute que la majorité voire, plus vraisemblablement, la totalité des " $C$. canis" de ces auteurs appartenaient à $C$. orientis ( $c f$. infra) qui, peutêtre, ne leur était pas connu. On peut rappeler que Klein (1971) note l'absence de C. canis au Cambodge, ce que sur quelques échantillonnages nous pensons pouvoir confirmer (J.C. B., non publié). Il semble en être de même dans le Nord-Laos (Beaucournu et al., 2001).

Rappelons que, à l'inverse des deux autres Ctenocephalides présents dans la sous-région indochinoise, C. canis est une puce sténoxène des canidés.

\footnotetext{
${ }^{5}$ Le nom entre parenthèses est celui qui est considéré comme valable par Musser et Carleton (in: Wilson et Reeder, 1993). Les sousespèces ne sont pas prises en compte.

${ }^{6}$ Par matériel examiné, nous entendons tous les exemplaires provenant du Vietnam ( $\mathrm{y}$ inclus ceux ne provenant pas de nos récoltes) vérifiés par l'un d'entre nous (J.C. B.) ou présents dans ses collections.
}

Nous apportons ici une station au Vietnam pour cette puce d'origine paléarctique.

Matériel examiné : 43, Nha Trang, sur chien, sans date (vers 1998).

Ce chien amené de Russie, était au Vietnam depuis au moins deux ans. Il est donc certain que plusieurs générations de puces se sont succédées sous ce climat, dans l'hypothèse où elles furent "importées" avec lui ou qu'il ne se contamina pas sur place.

\section{3 - Ctenocephalides felis felis (Bouché, 1835)}

Hérivaux et Toumanoff (1948a, b) sont, nous semble$\mathrm{t}$-il, les premiers à signaler cette espèce du Vietnam, à Saïgon-Cholon; nous avons vu que leurs relevés réunissaient au moins deux puces différentes, C. felis et $C$. orientis et nous ne pouvons donc en faire état. On peut toutefois noter que ces espèces ont une écologie apparemment analogue et la même euryxénie qui les fait se rencontrer sur chiens, chats, bovins, caprins, ovins, homme et même, accidentellement, sur les rats synanthropes. Dans les captures sur rongeurs et musaraigne (Suncus murinus) domiciliaires, Ctenocephalides spp. représentent, d'après les données de ces auteurs, 527 exemplaires sur 5587 puces; dans les prélèvements effectués directement sur le sol des habitations, ce chiffre monte à 6552 sur 6830 ! Ceci n'est pas sans évoquer les pseudo "invasions domiciliaires" de $C$. felis en Europe et aux États-Unis entre autres, liées à la présence même temporaire, d'un chien ou d'un chat dans l'habitation.

Hopkins et Rothschild (1953) la citent du Tonkin. Suntsov et al., (1992a, 1997) la signalent comme abondante dans les maisons et sur les animaux domestiques, particulièrement chiens et chats mais elle n'a pas été collectée sur les rongeurs commensaux ou les petits mammifères sauvages.

Matériel examiné : 85, des trois régions du Vietnam, dans des récoltes étalées de 1945 (in coll. J.C. B.) à 2000 .

4 - Ctenocephalides orientis (Jordan, 1925)

Nous renvoyons aux deux espèces précédentes pour les problèmes posés par l'identification de cette puce. Les caractères discriminants (chétotaxie céphalique et tibiale, phallosome) ont été revus récemment par Beaucournu et Ménier (1998) et Ménier et Beaucournu (1998)

D'après les collectes que nous avons étudiées, cette puce prédomine très nettement au Vietnam sur C. felis, seul autre Ctenocephalides euryxène nous l'avons dit. Ceci est normal puisque $C$. orientis est endémique de la région orientale, alors que $C$. felis y fut importé à une époque que nous ne pouvons préciser mais qui est, peut-être, historiquement ancienne (Beaucournu et al., 2000).

Dans la littérature, cette puce est citée du Vietnam par : 
- Hopkins et Rothschild (1953) : Annam, sur chien, et Tonkin, sur Macaca mulatta (Zimmermann, 1780) 7 . - Rostigayev et Grokovskaya (1966) qui la récoltent sur Rattus flavipectus ( $=R$. tanezumi) et $R$. exulans (Peale, 1848) au Nord-Vietnam.

Matériel examiné : 497, de toutes les régions du pays, uniquement en zones anthropisées, ce qui est étonnant étant donné son peu de spécificité et son eurytopie.

\section{Xenopsyllinae}

5. - Xenopsylla cheopis cheopis (Rothschild, 1903) Le foyer originel de cette puce est la vallée du Nil aux frontières de l'Ethiopie selon Jordan et Rothschild (1908). Ceci a été repris par Traub (1963, 1972d) qui donne le genre Arvicanthis comme hôte primitif; Beaucournu (1995) admet cette opinion en soulignant qu'au sud de cette zone existe la sous-espèce $X$. cheopis bantorum Jordan, 1938 (Schwan, 1992) et qu'au nord, sur l'île de Chypre, est l'endémique et espèce-jumelle X. acomydis Peus, 1977. Il parait d'ailleurs vraisemblable que la forme primitive soit bantorum, la zone afrotropicale étant le berceau du groupe cheopis, donnant par migration naturelle vers le Nord, cheopis s. sto. qui a eu l'heureuse opportunité, pour elle, de croiser les migrations vers l'Ouest du Rat noir et de se répandre avec lui dans toutes les zones compatibles avec son écologie : en effet, $X$. cheopis est plus sténotope que son hôte de capture.

Tous les travaux sur la peste au Vietnam signalent cette espèce : Cadet $(1917)^{8}$, Hérivaux et Toumanoff (1948a, b), Rostigayev et Grokovskaya (1966), Cavanaugh et al. (1968, 1969), Olson (1969), Velimirovic (1973), Suntsov et al. (1992a, b, 1995, 1997, 1999), Beaucournu et al. (2000); Adler et al. (2001)... Il est évident que seule la forme nominative est concernée.

Les hôtes primaires mais non primitifs (cf. inter alia Traub, 1963) de X. cheopis cheopis sont les Rongeurs Muridae appartenant essentiellement à l'ancien genre Rattus éclaté en sous-genres puis en genres à part entière ( $c f$. en particulier Musser et Carleton, 1993). Au Vietnam, on cite $R$. rattus (L., 1758), $R$. norvegicus (Berkenhout, 1769), R. exulans, $R$. tanezumi, R. nitidus (Hodgson, 1845), Mus cervicolor Hodgson, 1845, Bandicota indica (Bechstein, 1800), B. savilei Thomas, 1916. Un autre petit mammifère, insectivore cette fois, est d'autant plus fréquemment cité qu'il est souvent commensal des habitations : la musaraigne Suncus murinus. Les autres hôtes sont accidentels et on ne

\footnotetext{
${ }^{7}$ Il s'agit théoriquement d'une autre espèce : $M$. arctoides (I. Geoffroy, 1831), M. assamensis (M'Clelland, 1840) ou M. fascicularis (Raffles, 1821) : cf. Groves in Wilson et Reeder, 1993. Beaucournu et Jouan (non publié) viennent de retrouver cette puce sur un Macaca sp. en semi-liberté dans un village du Nord-Laos. La contamination d'origine canine semble ici évidente.

${ }^{8}$ Lasnet (1928), Gaide et Bodet (1930), Velimirovic (1972) ne font qu'exposer ou commenter les travaux de leurs devanciers.
}

peut même pas les considérer comme hôtes secondaires; l'homme en fait partie.

Il nous semble inutile de donner la liste des captures : Hérivaux et Toumanoff (op. cit.) en ont collecté plus de 5000 à Saïgon-Cholon! Deux points sont, à notre avis, à souligner :

- Sur les rongeurs synanthropes, le pourcentage de $X$. cheopis, par rapport aux autres espèces, est toujours très élevé : $95 \%$ pour Cadet, 53 à $100 \%$ pour Hérivaux et Toumanoff, $75 \%$ pour Rostigayev et Grokovskaya, supérieur à $99 \%$ pour Velimirovic de même que pour Cavanaugh et al., $100 \%$ pour Olson, $82 \%$ pour Suntsov et al. (1997). Le fait le plus important a, toutefois, été soulevé par Suntsov et al. (1992b, 1997): cette puce est liée avec ses hôtes aux zones anthropisées : à plus de $600 \mathrm{~m}$ des habitations X. cheopis disparait?. Ceci est d'un très grand intérêt épidémiologique et signifie l'existence de puces-relai entre rongeurs synanthropes et rongeurs selvatiques là où ces derniers sont impliqués dans l'enzootie pesteuse.

- Le deuxième fait marquant, et nous y reviendrons à propos de l'espèce suivante, est que la preéminence de $X$. cheopis, peut cacher la présence d'espèces congénériques ( $X$. astia ou $X$. vexabilis par exemple).

Matériel examiné : 27, toutes provenant de cases (ou paillottes)

\section{6 - Xenopsylla astia Rothschild, 1911}

Bien que considérée comme mauvais vecteur de la Peste humaine, cette espèce pose un problème quant à sa réelle fréquence au Vietnam. Elle n'a été signalée jusqu'à présent qu'en zones urbaines, et plus précisément portuaires, à notre connaissance. Beaucournu et al. (2000) estiment d'ailleurs que son berceau n'est pas oriental et, qu'en tous cas, celui-ci n'est sûrement pas la sous-région indochinoise.

L'existence de $X$. astia comme espèce autonome a été controversée au début des enquêtes, car pour un non spécialiste ce n'est qu'une Xenopsylla donc, a priori, $X$. cheopis! Cette réticence des épidémiologistes non entomologistes se retrouvera plus loin et peut expliquer les variations de fréquence observées, au Vietnam entre autres.

La première citation de cette puce pour ce pays fut faite par Fréville (1932), citant les déterminations de Souchard qui estime sa fréquence à $8 \%$ en Cochinchine; plus tard Hérivaux et Toumanoff (1948a, b) la trouvent en effectifs appréciables à Saïgon-Cholon, bien que nettement supplantée numériquement par $X$. cheopis : sur 6830 puces collectées dans les habitations (paillotes essentiellement), 133 appartiennent à cette espèce, 220 à X. cheopis, tout le reste étant Ctenocephalides spp. (cf. supra); sur 5588 puces collectées dans les mêmes zones sur hôtes (Rattus spp. et

\footnotetext{
${ }^{9}$ Suntsov et Suntsova (1999) repoussent cette limite à $1 \mathrm{~km}$.
} 
Suncus murinus), 232 sont $X$. astia, 527 Ctenocephalides spp., les autres sont $X$. cheopis (soit 4829). Si l'on ne considère que le genre Xenopsylla, $X$. astia suivant les conditions de prélèvement représente donc pour ces auteurs de 4,6 à $37,7 \%$ de l'effectif.

Or, les travaux plus récents soit ignorent cette puce, soit la trouvent à des pourcentages très bas. Dans cette même zone de Saïgon, Vélimirovic (1973) estime qu'il y a, au plus, $1 \%$ de $X$. astia, le reste étant exclusivement $X$. cheopis mais il faut relever que, d'une part, ne furent étudiées que les puces prélevées sur rats (pour Hérivaux et Toumanoff, le plus gros pourcentage provenait de puces "piégées" sur le sol des habitations) et que, d'autre part, l'auteur précise "Therefore, it is accepted that all fleas combed (il faut évidemment lire "non combed"10) are cheopis, and they are only periodically checked for identity".

En ce qui nous concerne, il n'a été noté qu'un exemplaire (non vérifié) prélevé sur $R$. rattus dans le port de Da-Nang. Il ne semble pas toutefois qu'il convienne de mettre en doute les déterminations de Hérivaux et Toumanoff, ce dernier était d'ailleurs un bon entomologiste : l'un de nous (J.C. B) a pu examiner ${ }^{11}$ dans ce qui subsiste de la collection Toumanoff à l'Institut Pasteur de Paris, une préparation parfaitement identifiée de $X$. astia, provenant d'Indochine (en l'occurrence l'actuel Vietnam), sans plus de précisions. Notons que toutes les captures signalées proviennent de l'Annam et surtout de la Cochinchine. Elle n'est connue ni du Tonkin, ni de Chine (Beaucournu et al., 2000).

L'hôte préférentiel est apparemment Tatera indica (Hardwicke, 1807). On peut d'ailleurs relever que les répartitions du rongeur et de la puce sont presque superposables. Les rongeurs capturés par Hérivaux et Toumanoff n'ont malheureusement pas été vérifiés par un mammalogiste.

Matériel examiné : une femelle, citée ci-dessus, ex collection Toumanoff.

7 - Xenopsylla vexabilis Jordan, 1925

Suntsov et al. (1992a, b) ont signalé cette espèce au Vietnam, sous le nom de $X$. vexabilis hawaiiensis Jordan, 1932 qui, en fait, comme X.v. meseris Jordan, 1936 n'est qu'un synonyme de la forme nominative (Smit, 1965). Ce pays était nouveau pour cette puce. $X$. vexabilis est une espèce orientale et australienne dont la dispersion dans de nombreuses îles du Pacifique est le fait de l'homme. Elle est, à notre avis, originaire de la sous-région indochinoise dans les limites

\footnotetext{
${ }^{10}$ Ce lapsus noté ici à la page 541 de l'article de Velimirovic est malheureusement repris dans les commentaires du tableau 5, p. 545. Il est vrai que l'auteur n'est pas entomologiste.

${ }^{11}$ Grâce à l'amabilité du Professeur F. Rodhain (Écologie des systèmes vectoriels) et de Mme R. Villeret, responsable des collections de ce Service.
}

de celle-ci que précise Lekagul (1988), avec comme hôte primitif Berylmys berdmorei (Blyth, 1851). Elle a, secondairement, parasité des Rattus s. sto., comme $R$. rattus, $R$. exulans, le plus souvent synanthropes, et a pu ainsi être dispersée par les déplacements humains. En Asie continentale elle est signalée, d'Ouest en Est, de Thaillande (Sankasuwan et al., 1969; Lekagul, op. cit.), du Cambodge (Klein, 1971) et du Vietnam (Suntsov et al., op. cit.). B. berdmorei y est unanimement donné comme hôte électif par Traub (1972c), Lekagul (op. cit.) et Suntsov et al. (1992a, b, 1995, 1997).

Mis à part l'article de Sankasuwan et al. (op. cit.) qui identifient $X$. vexabilis lors de l'étude du typhus murin urbain en Thaïlande, toutes les autres mentions concernant cette espèce en zone continentale, en font une puce de forêt tropicale. Pour Suntsov et al. (op. cit.) elle représente $13 \%$ de toutes les puces collectées, toutes les récoltes provenant de ce type de biotope; l'hôte dominant est $B$. berdmorei (porteur de 348 puces de cette espèce sur 369 X.vexabilis capturées). Quelques autres spécimens proviennent de $B$. bowersi (Anderson, 1879), Maxomys surifer (Miller, 1900) et Leopoldamys sabanus (Thomas, 1887), tous Muridés.

Étant donné ce que l'on sait de cette espèce (en particulier son rôle à Hawaii) l'intérêt de cette puce est certain dans l'épidémiologie de la peste ne serait-ce que dans l'entretien du "maillon" selvatique. C'est pourquoi, nous l'avons incluse dans cette note.

Matériel examiné : 10, tous sur hôtes selvatiques.

\section{Famille Pygiopsyllidae}

Pygiopsyllinae

8 - Lentistivalius klossi (Jordan et Rothschild, 1922)

Décrite du Vietnam (Dalat) sous le nom de Pygiopsylla klossi, cette puce est transférée dans le genre Stivalius la même année, par les descripteurs eux-mêmes (1922b), puis dans Lentistivalius, dans Aviostivalius et, enfin, dans Sphaerolemopsyllus par des auteurs chinois (Gong, in litt., 30.I.1996 ${ }^{12}$ ). Nous suivons Mardon (1981) en la maintenant dans Lentistivalius et considérons les genres Aviostivalius et Sphaerolemopsyllus comme non justifiés.

Nous avons collecté cette puce du Nord (femelle seulement) comme du Sud-Vietnam, mais elle est également connue de l'Inde, du Myanmar ${ }^{13}$, de Thailande, du Cambodge, de Malaisie occidentale, de Sumatra et de Java. La sous-espèce bispiniformis (Li et Wang, 1958), dont l'un de nous (J.C. B.) a pu examiner un couple, n'est signalée que du sud de la Chine : seul

\footnotetext{
${ }^{12}$ Nous n'avons pu trouver trace de la publication correspondante. ${ }^{13}$ Le Myanmar (ex Birmanie) est un pays nouveau pour L. klossi : Carin Chebà, Yadò, sur Mus jerdoni (= Niviventer fulvescens), L. Fea rec. I.1888 (det. JCB) (Pr. R. Poggi, Museum de Gênes, Italie).
} 
l'apex du sternite IX du mâle aurait pu justifier cette sous-espèce. Ce caractère toutefois est variable : le dessin princeps de Jordan (in Jordan et Rothschild, 1922a) est plus proche de "bispiniformis" que celui de l'exemplaire, d'origine non précisée, figuré par Mardon (1981) pour L. k. klossi. Dans le matériel topotype de Pygiopsylla klossi (in coll. J.C. B.), récolté par les Drs J. Gaud en 1945 et R. Lumaret en 1955, un mâle est identique à celui dessiné par Jordan, un autre est proche de celui figuré par Mardon. Nous considérons donc bispiniformis comme synonyme de la forme nominative (syn. nov.).

Suntsov et al. (1992b, 1997) ont indiqué que cette puce présentait au Vietnam un intérêt épidémiologique particulier puisque c'est la seule qui, dominante en forêt tropicale, arrive à constituer jusqu'à $3 \%$ de la faune pulicidienne des villages et des zones agricoles environnantes. Ce vecteur est le seul, dans ces observations, à faire le lien entre mammifères selvatiques et synanthropes.

Ses hôtes sont assez variés, mais se recrutent presque uniquement chez les Muridés. Toutefois en ne tenant compte que des mammifères dûment identifiés, l'hôte préférentiel est au Vietnam (mais sur de faibles effectifs) Dremomys rufigenis (Blanford, 1878), un Sciuridé. Les autres rongeurs parasités sont, pour nos exemplaires, Maxomys surifer (Miller, 1900), Rattus exulans, R. nitidus, $R$. rattus, Bandicota savilei, Niviventer fulvescens, N. langbianis (Robinson et Kloss, 1922), N. cremoriventer (Miller, 1900), Berylmys berdmorei et Leopoldamys edwardsi (Thomas, 1822), tous des Muridés. Au total une quarantaine d'exemplaires furent récoltés. Matériel examiné : neuf.

\section{FAMILLE CERATOPHYLLIDAE $^{14}$}

\section{Leptopsyllinae}

9 - Leptopsylla segnis (Schönherr, 1811)

Cette puce, répandue dans la majeure partie du monde par la souris "domestique" Mus musculus L., 1758, ne figure pas dans nos récoltes. Aucun auteur ayant travaillé dans le sud du Vietnam ne la signale; Hérivaux et Toumanoff (1948a) insistent même sur ce fait pour la région de Saïgon-Cholon. La seule référence que nous connaissions (Rostigayev et Grokovskaya, 1966) concerne le Nord-Vietnam où ces auteurs notent que $13 \%$ de leurs captures de L. segnis proviennent de Rattus tanezumi, mais on ne connaît pas le nombre total d'individus récoltés, ni leur biotope.

Matériel examiné : néant ( $c f$. note 6).

\footnotetext{
${ }^{14}$ Acropsylla episema Rothschild, 1911 (Ceratophyllidae : Amphipsyllinae) semble fréquente sur les rongeurs commensaux de Chine méridionale. Au Vietnam, elle ne fut trouvée qu'une fois dans cette situation, à Dalat. C'est essentiellement une puce de Muridés, en général selvatiques.
}

Ceratophyllinae

10 - Nosopsyllus wualis Jordan, 1941

Bien que $N$. wualis soit signalé de plusieurs points du Nord-Vietnam par Rostigayev et Grokovskaya (1966) ${ }^{15}$, elle ne figure pas dans les récoltes étudiées.

Ses hôtes classiques sont des muridés, essentiellement synanthropes : au Vietnam, il s'agit de Rattus norvegicus et $R$. tanezumi.

Sa répartition connue est restreinte et ne concerne que le Sud de la Chine (terra typica) et le Nord-Vietnam. Matériel examiné : néant.

\section{CONCLUSION}

L es puces synanthropes les plus fréquentes au Vietnam sont, comme dans toute l'Asie continen. $X$. astia est rare et, manifestement, en limite de répartition.

Une seule espèce, L. klossi, est connue comme pouvant faire le lien entre rongeurs synanthropes et selvatiques; cela confirme que la Peste est "récente" dans ce pays et, qu'en dépit de sa permanence en zones urbanisées, elle n'y constitue pas un foyer invétéré.

\section{RÉFÉRENCES}

Adler G.H., Suntsova N.I., Suntsov V.V. \& Mangan S.A. Fleas (Siphonaptera) collected from small mammals in Southern Viet Nam in 1997-1998. Joumal of Medical Entomology, 2001, 38, 210-213.

Advisory Committee. Reports on plague investigations in India. Journal of Hygiene, 1907, 7, 323-476.

Beaucournu J.C. À propos du vecteur de la peste en Europe occidentale au cours de la deuxième pandémie. Bulletin de la Société Française de Parasitologie, 1995, 13, 233-252.

Beaucournu J.C., Jouan R. \& Ménier K. Insectes ectoparasites du chien au Laos. Revue de Médecine Vétérinaire, 2001, 152, 77-82.

Beaucournu J.C. \& Launay H. Les Puces (Siphonaptera) de France et du bassin méditerranéen occidental. In : Faune de France - 76, Fédération française des Sociétés de Sciences Naturelles éditeur, 1990, 548 pp.

Beaucournu J.C., Le Piver M. \& Guiguen C. Actualité de la conquête de l'Afrique intertropicale par Pulex irritans Linné, 1758. Bulletin de la Société de Pathologie Exotique, 1993, 86, 290-294.

Beaucournu J.C. \& Ménier K. Le genre Ctenocephalides Stiles et Collins, 1930 (Siphonaptera, Pulicidae). Parasite, 1998, 5, 3-16.

${ }^{15}$ wualis wualis ou wualis leizhonensis Li, Huang et Liu, 1986. 
Beaucournu J.C. \& Sountsov V.V. Puces du genre Neopsylla collectées au Vietnam : description de $N$. bana n. sp. (Siphonaptera, Ctenophthalmidae). Bulletin de la Société entomologique de France, 1997, 102, 205-209.

Beaucournu J.C. \& SOunTSOV V.V. Un Ctenophthalmus nouveau de la région orientale (Siphonaptera, Ctenophthalmidae). Bulletin de la Société entomologique de France, 1998a, 103, 33-35.

Beaucournu J.C. \& Sountsov V.V. Liuopsylla simondi n. sp., puce nouvelle du Vietnam (Siphonaptera, Ctenophthalmidae, Liuopsyllinae). Bulletin de la Société entomologique de France, 1998b, 103, 209-212.

Beaucournu J.C. \& Sountsov V.V. Puces nouvelles ou peu connues du Vietnam. Ctenophthalmidae et Pygiopsyllidae (Siphonaptera). Bulletin de la Société entomologique de France, 1999a, 104, 77-83.

Beaucournu J.C. \& Sountsov V.V. Puces nouvelles ou peu connues du Vietnam. Ceratophyllidae (Siphonaptera). Bulletin de la Société entomologique de France, 1999b, 104, 447-453.

Beaucournu J.C., Sountsov V.V., LY Thi Vi Huong \& Sountsova N.I. Esquisse d'une biogéographie des puces (Insecta, Siphonaptera) du Vietnam. Biogeographica, 2000, 76, 120.

Cadet G. La Peste du Sud-Annam. Bulletin de la Société de Pathologie exotique, 1917, 10, 41-65.

Cavanaugh D.C., Hunter D.H., Nguyen-Van-Ba, Tran-CongDung, Ryan P.F. \& Marshall J.D. Ecology of plague in Vietnam III. Sylvatic plague; Bandicota indica, a transitional species. Transactions of the Royal Society of Tropical Medicine and Hygiene, 1968, 62, 456.

Cavanaugh D.C., Ryan P.F. \& Marshall J.D.The role of commensal rodents and their ectoparasites in the ecology and transmission of plague in Southeast Asia. Bulletin Wildlife Disease Association, 1969, 5, 187-194.

FrÉville ${ }^{16}$. Contribution à l'épidémiologie de la peste en Cochinchine. Annales de Médecine et de Pharmacie coloniales, 1932, 30, 653-679.

GAIDE $^{16}$ \& BODET ${ }^{16}$. La peste en Indochine. Imprimerie d'ExtrêmeOrient, Hanoï, 1930, 33 pp.

Hérivaux A. \& Toumanoff C. Épidémiologie de la peste à Saïgon-Cholon (1943). L'étude de la faune pulicidienne des rats dans ses rapports avec la transmission de la peste. Bulletin de la Société de Pathologie Exotique, 1948, 41, 47-59.

Hérivaux A. \& Toumanoff C. Étude de la "faune pulicidienne domiciliaire" des rats au cours d'une épidémie de peste à Saïgon; ses conséquences pratiques. Bulletin de la Société de Pathologie Exotique, 1948, 41, 318-325.

Hopkins G.H.E. \& Rothschild M. An illustrated Catalogue of the Rothschild collection of fleas (Siphonaptera) in the British Museum (Natural History) ; Vol. I : Tungidae and Pulicidae. British Museum, 1953, 361 pp.

JORDAN K. Three new species of Neopsylla (Siphonaptera) from the Oriental regions. Novitates Zoologicae, 1931, 36, 220-2224.

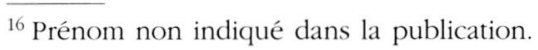

JORDAN K. Siphonaptera collected by Dr. Gerd Heinrich in Burma. Novitates Zoologicae, 1939, 41, 362-375.

Jordan K. \& RothsChILD N.C. Revision of the non combed eyed Siphonaptera. Parasitology, 1908, 1, 1-100.

JordAN K. \& ROTHSCHILD N.C. New species of Siphonaptera collected by Mr. C. Boden Kloss in the Malay Peninsula and South Annam. Ectoparasites, 1922a, 1, 21-222.

Jordan K. \& Rothschild N.C. On Pygiopsylla and the allied genera of Siphonaptera. Ectoparasites, 1922b, 1, 231-265.

KLEIN J.M. La faune des puces du Cambodge (Siphonaptera). Cabiers de l'ORSTOM, série Entomologie médicale-Parasitologie, 1971, 9, 223-238.

LASNET $^{16}$. Indochine française, in Rongeurs et Puces dans la conservation et la transmission de la peste. Masson et Cie éd., Paris, 1928, 212-220.

LeKagul. B. Mammals of Thailand. Kurusapha Ladprao ( 2 ème edition, 1988, Darnsutha Press, LI + 758 pp.).

LEwIS R.E. Résumé of the Siphonaptera (Insecta) of the World. Journal of Medical Entomology, 1998, 35, 377-389.

Ménier K. \& Beaucournu J.C. Taxonomic Study of the Genus Ctenocephalides Stiles \& Collins, 1930 (Insecta: Siphonaptera: Pulicidae) by using Aedeagus Characters. Journal of Medical Entomology, 1998, 35, 883-890.

Olson W.P. Rat-flea indices, Rainfall, and Plague outbreaks in Vietnam, with emphasis on the Pleiku area. The American Journal of Tropical Medicine and Hygiene, 1969, $\mathrm{n}^{\circ} 18,621-628$.

OMS. Relevé épidémiologique hebdomadaire. La Peste humaine en 1994. 1996, n $71,165-172$.

OMS. Relevé épidémiologique hebdomadaire. La Peste humaine en 1995. 1997, n 46, 344-347.

OMS. Relevé épidémiologique hebdomadaire. La Peste humaine en 1998 et 1999. 2000, $\mathrm{n}^{\circ} 42,338-343$.

Rostigayev B.A. \& Grokovskaya I.M. (On the flea-fauna in North Viet-nam). Zoologicheskiy Zhurnal, Moscou, 1966, 45, 61-67 (en russe, résumé anglais).

Sankasuman V., Pongrradit P., Bodhidatta P., ThonglonGYA K. \& Winter P.E. Murine typhus in Thailand. Transactions of the Royal Society of Tropical Medicine and Hygiene, 1969, 63, 639-643.

Schwan T.G. Xenopsylla bantorum is an East African Subspecies of $X$. cheopis (Siphonaptera : Pulicidae). Journal of Parasitology, 1992, 29, 927-993.

SмIT F.G.A.M. Siphonaptera of New Zealand. Transactions of the Royal Society of New Zealand (Zoology), 1965, 7, 150.

Suntsov V.V., LY Thi Vi Huong \& Suntsova N.I. (Notes on fleas (Siphonaptera) in plague nidi on Tay Nguyen plate (Vietnam)) (en russe, résumé anglais). Parazitologia, 1992a, 26, 516-520.

Suntsov V.V., LY Thi Vi HuOng \& Suntsova N.I. (Some features of the flea (Insecta, Siphonaptera) fauna of small mammals in Vietnam) (en russe, résumé anglais). Zoologischesliy Zhumal, 1992b, 71, 88-94.

SunTsov V.V., LY Thi Vi Huong \& Suntsova N.I. (The role of wild small animals in the plague foci of Vietnam) (en russe, résumé anglais). Zoologischesliy Zhurnal, 1995, 74, 119-127. 
Suntsov V.V., LY Thi Vi Huong, Suntsova N.I. \& Gratz H.G. Plague foci in Vietnam: zoological and parasitological aspects. Bulletin of the World Health Organization, 1997, 74, 117-123.

Suntsov V.V. \& Suntsova N.I. Fleas (Siphonaptera) and Gamasine mites (Gamasina) of small mammals on territories of "Ecocide" and intensive economic development in South Vietnam. Entomological Review, 1999, 79, 606-612 (traduit du Zoologicheskii Zhurnal, 1999, 78).

Traub R. The fleas of Egypt. Two new fleas of the genus Nosopsyllus Jordan, 1933. Proceedings of the Entomological Society of Washington, 1963, 65, 81-97.

Traub R. Notes on fleas and the ecology of plague. Journal of Medical Entomology, 1972, 9, 603.

Traub R. The Gunong Benom expedition 1967. 11. Notes on zoogeography, convergent evolution and taxonomy of fleas (Siphonaptera), based on collections from Gunong Benom and elsewhere in south-east Asia. I. New taxa (Pygiopsyllidae, Pygiopsyllinae). Bulletin of the British Museum (Natural History), 1972, 23, 201-305.

Traub R. The Gunong Benom expedition 1967. 12. Notes on zoogeography, convergent evolution and taxonomy of fleas (Siphonaptera), based on collections from Gunong Benom and elsewhere in south-east Asia. II. Convergent evolution. Bulletin of the British Museum (Natural History), 1972, 23, 307-387.

Traub R. The Gunong Benom expedition 1967. 13. Notes on zoogeography, convergent evolution and taxonomy of fleas (Siphonaptera), based on collections from Gunong Benom and elsewhere in south-east Asia. III. Zoogeography. Bulletin of the British Museum (Natural History), 1972, 23, 389-450.

Velimirovic B. Plague in south-east Asia. A brief historical summary and present geographical distribution. Transactions of the Royal Society of Tropical Medicine and Hygiene, 1972, 66, 479-504.

Velimirovic B. Surveillance of small mammals and flea Vectors in a plague-infected port. Zeitschrift für Tropenmedizin und Parasitologie, 1973, 24, 539-548.

WiLSON D.E. \& REEDER D.A.M. Mammal species of the World, a taxonomic and geographic reference. 2 nd edition, Smithsonian Institution Press, Washington, 1993, 1207 pp.

Yersin A. L'épidémie de peste à Nha-Trang de juin à octobre 1898. Annales d'Hygiène et de Médecine Coloniales, 1899, 2, 378-390.

Reçu le 13 juin 2001

Accepté le 15 octobre 2001 